\title{
Influence of the Morphology of Lysozyme-Shelled Microparticles on the Cellular Association, Uptake, and Degradation in Human Breast Adenocarcinoma Cells
}

\author{
Francesca Cavalieri, Marisa Colone, Annarita Stringaro*, Mariarosaria Tortora, \\ Annarica Calcabrini, Meifang Zhou, and Muthupandian Ashokkumar*
}

The ultrasound-assisted self-assembly and cross-linking of lysozyme at the water-air and water-perfluorohexane interfaces are shown to produce lysozyme-shelled hollow microbubbles (LSMBs) and microcapsules (LSMC), respectively. The arrangement of lysozyme at the air-liquid or oil-liquid interfaces is accompanied by changes in the bioactivity and conformational state of the protein. The interaction of LSMB and LSMC with human breast adenocarcinoma cells (SKBR3) is studied. LSMB and LSMC are phagocyted by cells within $2 \mathrm{~h}$ without exerting a cytotoxic activity. The cellular internalization kinetics of LSMB and LSMC and the effects on cell cycle are evaluated using flow cytometry. Evidence for the internalization of microparticles and degradation within the cell are also monitored by confocal and scanning electron microscopic analyses. The integrity of cell membrane and cell cycle is not affected by LSMBs and LSMCs uptake. These studies show that the positively charged LSMB and LSMC are not cytotoxic and can be readily internalized and degraded by the SKBR3 cells. LSMBs and LSMCs show a different uptake kinetics and intracellular degradation pattern due to differences in the arrangement of the protein at the air-liquid or oil-liquid interfaces.

minimizing damage to normal cells. To pursue this approach, ${ }^{[2]}$ biomaterial science has stepped into the formulation of smart materials and miniaturized drug delivery devices. There is an increasing arsenal of nano-microplatforms under evaluation for therapeutic applications including polymeric micelles and capsules, liposomes DNA, and protein-based micro-nanoparticles. ${ }^{[3-5]}$ By using both passive (enhanced permeability and retention effect) and active targeting strategies, micro-nanocarriers can deliver a high dose of drugs in cancer cells while minimizing toxicity in normal cells. ${ }^{[6]}$ Alternatively, a remote and external control of drug delivery is possible where the micro-nanocarrier is responsive to an applied stimulus such as infrared light,, ${ }^{[7]}$ ultrasound, ${ }^{[8]}$ magnetic, or electric field..$^{[9]}$ A promising strategy to remotely target cells and organs is to develop ultrasound-responsive micronanocarriers that release their drug payload

\section{Introduction}

The application of nanomedicine for cancer therapy has received considerable attention in recent years. ${ }^{[1]}$ The key issue is to achieve the desired concentration of therapeutic agents in tumor sites, thereby destroying cancerous cells while

Dr. F. Cavalieri, Dr. M. Zhou, Prof. M. Ashokkumar School of Chemistry

The University of Melbourne

Parkville, Melbourne

Victoria, 3010, Australia

E-mail:masho@unimelb.edu.au

Dr. F. Cavalieri, Dr. M. Tortora

Dipartimento di Scienze e Tecnologie Chimiche

Università di Roma Tor Vergata

00173 Roma, Italy

Dr. M. Colone, Dr. A. Stringaro, Dr. A. Calcabrini

Dipartimento di Tecnologie e Salute,

Istituto Superiore di Sanità, 00161 Roma, Italy

E-mail: annarita.stringaro@iss.it

Prof. M. Ashokkumar

Chemistry Department

King Abdulaziz University

Jeddah, Saudi Arabia only in response to an acoustic stimulus. ${ }^{[10-12]}$ Microbubbles (MBs) are gas-filled colloidal particles generally coated with a protein, lipid, or a surfactant layer with a size range between $0.5-10 \mu \mathrm{m} .{ }^{[13]}$ In the past, MBs have been used in clinical practice as ultrasound contrast agents in ultrasound myocardial perfusion imaging ${ }^{[10]}$ and focal liver lesion diagnosis and recently approved for the detection of focal breast lesions. ${ }^{[14,15]}$ During the last decade, MBs have been widely investigated for the innovative therapeutic interventions. ${ }^{[16]}$ For instance, it was recently shown that ultrasound-mediated MB vascular disruption can enhance tumor responses to radiation in vivo. ${ }^{[17]}$ Indeed, ultrasound has a number of attractive features as a drug delivery modality. Ultrasonic irradiation of tissue with a millimeter precision is feasible and ultrasound beam may be directed toward deeply located body sites in precise energy deposition patterns. Both gas and perfluorocarbon (PFC)-filled microparticles are highly suited as ultrasound contrast agents in clinical applications. ${ }^{[18]}$ The acoustic impedance of gas and PFC compared with blood and solid tissue enables the MBs and PFC microcapsules (MCs) with echogenic properties in biological tissues. Lipidcoated PFCs nano- and microdroplets have also been used as delivery vehicles. ${ }^{[19,20]} \mathrm{PFCs}$ are biologically inert, for this reason they have been used for intravascular oxygen transport, ${ }^{[21]}$ ophthalmology, ${ }^{[22]}$ and lung surfactant replacement preparations. ${ }^{[23]}$

DOI: 10.1002/ppsc.201300025 
Generally, lipophilic drugs are deposited in the lipid monolayers and a major concern in the development of injectable PFCwater emulsions is to counteract Ostwald ripening, ${ }^{[24]}$ which is the main mechanism responsible for particle growth over time. Recently, polymer-shelled MBs and MCs have been synthesized by high-intensity ultrasound-induced emulsification and selfcross-linking of lysozyme and thiolated polymethacrylate in an aqueous solution without using any additional cross-linking agents. ${ }^{[25,26]}$ The ultrasonic methodology constitutes a platform technique offering versatility in the synthesis of relatively monodisperse air-filled and perfluorohexane (PFH) microparticles where the shell material can be a thiolated protein or synthetic polymer. ${ }^{[26,27]}$ Particularly, the ability to synthesize nanobubbles $^{[28]}$ has opened new opportunities to deliver therapeutic agents that require targeted extravasation from blood vessels into the tissues crossing the epithelial barriers.

To the best of our knowledge, an ultrasonic synthesis of lysozyme-shelled hollow particles is the only method that allows control over size and size distribution of lysozyme micro-nanoparticles. Conventional techniques for producing micrometer-sized protein particles include grinding, jet milling, liquid-phase antisolvent precipitation, freeze-drying and spray drying, and chemical cross-linking by glutaraldehyde. ${ }^{[29,30]}$ Unfortunately, these processes often incur thermal and chemical degradation and involve the use of organic solvents and toxic reagents resulting in high levels of cytotoxic residues, inter-batch particle size variability, broad size distribution, and particle irregular shapes. The thick $(150 \mathrm{~nm})^{[27]}$ and covalently cross-linked protein shell of lysozyme-shelled microbubbles (LSMB) prevents bubbles and oil droplets from coalescence and imparts to the particles a remarkable stability (up to 1 year). Particularly for LSMBs, the encapsulating shell is necessary to sustain the gas cavity and to reduce both the diffusion of gas leaving the core and the surface tension, as modeled in a modified Epstein-Plesset equation. ${ }^{[31]}$ The multifunctional LSMBs and lysozyme-shelled microcapsules (LSMCs) may provide drug payload capacity and a large surface for conjugation of targeting ligands. In addition, LSMCs offer the opportunity to employ a unique magnetic resonance imaging (MRI) signature deriving from its fluorine $\left({ }^{19} \mathrm{~F}\right)$ core. ${ }^{[32]}$ When combined with local drug delivery, the ${ }^{19} \mathrm{~F}$ signal serves as a highly specific marker for the quantitative assessment of drug dosing. ${ }^{[18]}$

Here, we report on the interaction of LSMBs and LSMCs with human breast adenocarcinoma cells (SKBR3). The kinetics of uptake, intracellular degradation, and induction of cytotoxicity of LSMBs and LSMCs have been evaluated. The structural and functional properties of lysozyme assembled into micrometer-sized particles, LSMBs and LSMCs, have been extensively studied and correlated to the cellular uptake and degradation behavior of microparticles.

\section{Results and Discussion}

\subsection{Comparison of Structural and Functional Properties of LSMB and LSMC}

The physical and chemical properties of LSMBs and LSMCs play an important role in determining the interactions with SKBR3 cells. The particle size, surface charge, and surface chemistry of LSMBs and LSMCs determine the cellular entry mechanisms and intracellular trafficking patterns. ${ }^{[3-35]}$ The ultrasonic synthetic method of LSMBs and LSMCs has been previously discussed. ${ }^{[25-28]}$ In brief, there are three processes (Scheme 1) involved: i) the emulsification of the gas or oil to

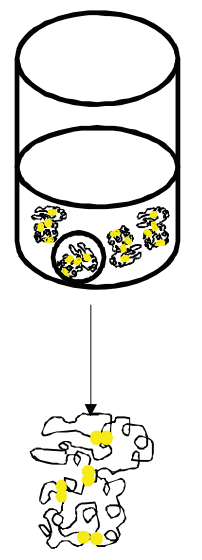

Native lysozyme

\section{Disulfide bonds \\ perfluorohexane \\ air}
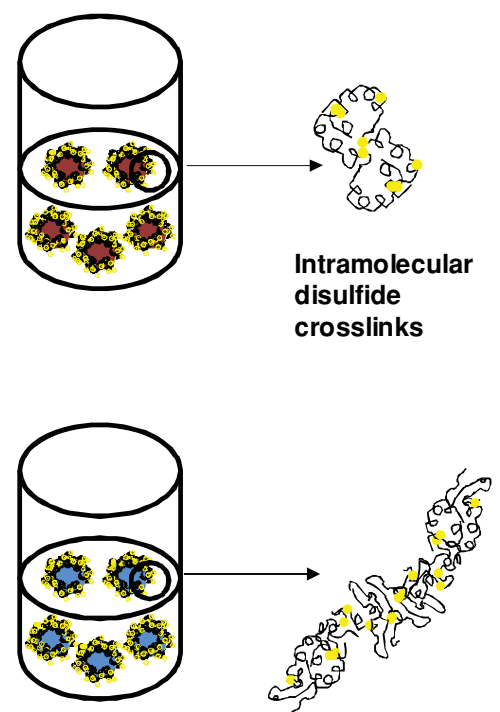

Intermolecular disulfide crosslinks

Scheme 1. Schematic illustration showing the LSMB and LSMC preparation technique through ultrasound-induced interfacial cross-linking of partially denatured lysozyme. 

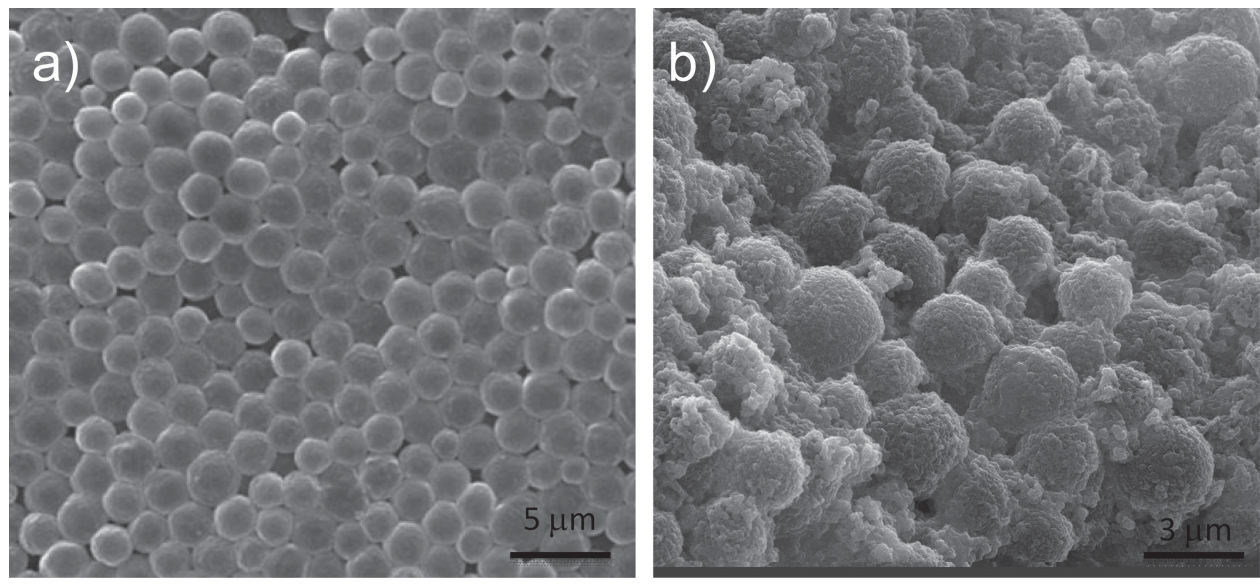

Figure 1. SEM images of a) LSMBs and b) LSMCs.

form a suspension of MBs or microdroplets in an aqueous solution, ii) the aggregation of denatured lysozyme at the airwater or oil-water interface, iii) the chemical cross-linking of cysteine residues of the partially denatured lysozyme. The hydrophobic interaction between the partially denatured protein subunits is one of the requirements for the formation of LSMBs and LSMCs (Scheme 1). However, the control of size, polydispersity, and long-term stability of LSMBs and LSMCs require a further mechanism to stabilize the protein-shelled microparticles. The protein shell is stabilized by inter-protein disulfide cross-linking of cysteine residues by the superoxide radicals generated during the sonolysis of water. Scanning electron microscopy (SEM) images of resulting LSMBs and LSMCs are shown in Figure 1.

Making a comparison between the physico-chemical and functional properties of LSMBs and LSMCs, we observed some similarities and differences indicating a different arrangement of the protein at the air-liquid or oil-liquid interfaces (Table 1). The diameter measured by optical and electron microscopic techniques was about $2.5 \mu \mathrm{m}$ for both microparticles. The residual thiol content on LSMB shell was found to be significantly higher $\left(62 \mathrm{mmol} \mathrm{mg}^{-1}\right)$ than that on LSMC shell $\left(12 \mathrm{mmol} \mathrm{mg}^{-1}\right)$. The secondary protein structure of LSMBs and LSMCs was evaluated by circular dichroism analysis. CD spectra of LSMB indicated a conformational transition of lysozyme from the native-like conformation $(26 \% \alpha$ helix, $22 \% \beta$ strand, $52 \%$ random coil) to a secondary structure rich in $\beta$-sheets (13\% $\alpha$ helix, 39\% $\beta$ strand, $49 \%$ random coil) (Figure 1a, Supporting Information). Conversely, the lysozyme secondary structure in LSMC exhibited an increase in helical and $\beta$ - sheet content ( $32 \alpha$ helix, $30 \beta$ strand, 38 random coil). To further evaluate and compare the structural properties of LSMBs and LSMCs, time-resolved fluorescence measurements were carried out.

Lysozyme contains six tryptophans. Time-resolved fluorescence measurements $\left(\lambda_{\mathrm{ex}}=298 \mathrm{~nm}, \lambda_{\mathrm{em}}=340 \mathrm{~nm}\right)$ indicated a double exponential decay of $3.9 \mathrm{~ns}$ and $1.3 \mathrm{~ns}$ for LSMB and $3.3 \mathrm{~ns}$ and $1.3 \mathrm{~ns}$ for LSMC. These fluorescence decay values were not significantly different from those measured for the native lysozyme, $3.1 \mathrm{~ns}$ and $0.9 \mathrm{~ns}$. Degradation of LSMBs and LSMCs by a protease was assessed by monitoring the optical density decrease as a function of time

Table 1. Structural and functional properties of LSMB and LSMC.

\begin{tabular}{|c|c|c|}
\hline Structural and functional property & LSMC & LSMB \\
\hline Diameter [um] & $2.5 \pm 0.5$ & $2.5 \pm 0.5$ \\
\hline$\xi$ potential Milli-Q [mV] & $+29 \pm 4$ & $+32 \pm 2$ \\
\hline$\xi$ potential, $15 \times 10^{-3} \mathrm{M} \mathrm{NaCl}[\mathrm{mV}]$ & $-22 \pm 8$ & $+22 \pm 0.4$ \\
\hline$\xi$ potential after BSA coating in [Milli-Q] & $-30 \pm 3$ & $-24 \pm 8$ \\
\hline \multirow[t]{3}{*}{ Percentages of protein secondary structure } & $32 \alpha$ helix & $13 \alpha$ helix \\
\hline & $30 \beta$ strand & $39 \beta$ strand \\
\hline & 38 random coil & 49 random coil \\
\hline Thiols content $\left[\mathrm{mmol} \mathrm{g}^{-1}\right]$ & $12 \pm 2$ & $62 \pm 1$ \\
\hline Antimicrobial activity & No & Yes \\
\hline Proteolytic degradation & $100 \%$ degradation in $70 \mathrm{~min}$ & $100 \%$ degradation in $30 \mathrm{~min}$ \\
\hline \multirow[t]{2}{*}{ Fluorescence time decay of trypthophans } & $3.9 \mathrm{~ns}(43 \%)$ & $3.3 \mathrm{~ns}(44 \%)$ \\
\hline & $1.3 \mathrm{~ns}(57 \%)$ & $1.3 \mathrm{~ns}(55 \%)$ \\
\hline
\end{tabular}


(Figure1b, Supporting Information). The protease digestion of LSMBs was complete after 30 min treatment, whereas the degradation of LSMCs required more than $1 \mathrm{~h}$. The differences in degradation kinetics suggest that in LSMBs the partially denatured lysozyme is more susceptible to degradation than in LSMCs as the unfolding greatly favors access of the protease to the polypeptide chain. ${ }^{[36]}$ In addition, these results demonstrate the biodegradability of both LSMBs and LSMCs, ruling out the possible formation of amyloid-like fibrils within the lysozyme shell induced by the sonication process or chemical cross-linking. As lysozyme isoelectric point is 10, both LSMCs and LSMBs are positively charged colloidal particles. The $\zeta$-potential values in Milli-Q water were $+29 \mathrm{mV}$ and +32 mV for LSMCs and LSMBs, respectively. In $15 \times 10^{-3} \mathrm{~m}$ $\mathrm{NaCl}$ buffer, the surface charge of LSMBs decreased to $+22.4 \pm$ $0.5 \mathrm{mV}$ due to the shielding effect of salt. Differently, the surface charge of LSMCs turns to a negative value of $-23 \pm 2 \mathrm{mV}$, indicating a rearrangement of the protein at the interface induced by the increase of ionic strength. The conformational transition of the protein in the LSMCs at the oil-water interface promotes the exposure of the negatively charged residues toward the aqueous phase. The positively charged surface of LSMBs and LSMCs provides a template for the assembly of negatively charged proteins present in the blood or cell culture medium that largely controls the biological in vitro response of LSMBs and LSMCs. Bovine serum albumin (BSA, the most abundant blood plasma protein) readily associates with both types of microparticles. The $\zeta$-potential of LSMBs and LSMCs incubated with BSA changes to negative values, $-24 \pm 8 \mathrm{mV}$ and $-30 \pm 3 \mathrm{mV}$, respectively, indicating the effective adsorption of BSA. The driving force behind BSA adsorption must be a combination of electrostatic, hydrophobic, and thiols interactions. Based on the above discussion, we speculate that, to form LSMBs, lysozyme assembles at the air-liquid interface and form a hydrogel-like structure stabilized by both newly formed intermolecular disulfide crosslinks and $\beta$-sheet domains (Scheme 1). Due to the disulfide interchain linkages, the denatured lysozyme in LSMBs is not able to reconstitute the fully native structure, the unreacted - SH groups remaining free and accessible. A different mechanism can be proposed for LSMC formation, where the interaction between PFH and the hydrophobic domain of the reduced lysozyme promotes protein refolding. Fluorinated organic solvents, such as trifluorethanol (TFE) and hexafluoro-propan-2-ol, ${ }^{[37,38]}$ are known to be very strong helix-inducing cosolvents and effective agents for the structural stabilization of protein secondary structures. A similar effect was reported for lysozyme where the helical content increases in the presence of TFE, by the extension of existing $\alpha$-helices, by the induction of new $\alpha$-helices, or by an increase in the regularity of the existing helices. ${ }^{[39]}$ During ultrasoundassisted formation of LSMCs, intramolecular disulfide bonds constitute concomitantly with protein refolding and restructuring at the oil-liquid interface, resulting in protein-shelled MCs stabilized mainly by hydrophobic interactions rather than intermolecular cross-linking. The absence of intermolecular protein cross-linking via disulfide bonds is also corroborated by the greater conformational freedom of lysozyme observed in LSMCs. However, not all four native disulfide bridges are recovered and some sulfhydryl groups $\left(12 \mathrm{mmol} \mathrm{g}^{-1}\right)$ are still present in the LSMC shell. The similarity between lysozyme LSMCs and LSMBs fluorescence decay times indicates that tryptophan residues in the protein experience similar solvent and hydrophobicity environment. This suggests that the hydrophobic core of protein, where tryptophans are present, is not affected by microparticle formation. Hence, the structural properties of LSMBs and LSMCs suggest a different rearrangement of lysozyme as a function of the chemical nature of the microparticle core. Lysozyme is an effective antimicrobial agent. ${ }^{[3]}$ The antimicrobial activities of LSMBs and LSMCs were evaluated and compared observing the disruption of Micrococcus lysodeikticus cell wall. We have previously shown that LSMBs possess significant antimicrobial activity. ${ }^{[11,25]}$ Conversely with LSMCs, the integrity of M. lysodeikticus cells was not affected indicating that LSMCs do not bear any bactericidal activity. Partially unfolded lysozyme is reported to be a potent bactericidal agent ${ }^{[36]}$ against both Gram-negative (Escherichia coli) and Gram-positive (Staphylococcus aureus, Micrococcus luteus) bacteria regardless of its enzymatic muramidase activity. The antimicrobial activity of LSMBs can be ascribed to a residual muramidase activity and to the surface hydrophobic moieties exposed upon protein unfolding and cross-linking. These hydrophobic patches can promote the interaction of LSMBs with the bacterial membrane, compromising its integrity and functions. The lack of antimicrobial activity in LSMCs indicates a more hydrophilic surface and absence of muramidase activity.

\subsection{Effect of Microbubbles and Microcapsules on Human Breast Adenocarcinoma Cells Viability}

To determine whether the different structural features of microparticles can affect their biological response, the interaction of LSMBs and LSMCs with SKBR3 was first assessed in terms of in vitro toxicity. The LSMBs and LSMCs are designed to maintain integrity in the bloodstream and in the extracellular environment and subsequently disassemble in the intracellular environment due to the enzymatic degradation. The stability of LSMBs and LSMCs in cell culture medium was verified. Both LSMBs and LSMCs retain their colloidal stability and do not leak gas or oil in the medium after few hours of incubation (data not shown). SKBR3 cell viability was studied at an LSMB- and LSMC-to-cell ratio ranging from 25 to 100 for $24 \mathrm{~h}$ by MTT, (3-(4,5-dimethylthiazol-2-yl)-2,5-diphenyltetrazolium bromide assay, which measures mitochondrial metabolic activity. As shown in Figure 2, the percent control proliferation was not affected after incubation with both LSMBs and LSMCs at up to $100 \mathrm{MB}$ or $\mathrm{MC} /$ cell for $24 \mathrm{~h}$ and $48 \mathrm{~h}$, while it slightly decreased to $75-80 \%$ after $72 \mathrm{~h}$. On the basis of this evidence, both MBs and MCs could be considered to induce negligible toxicity to SKBR3 cells.

\subsection{Cell Membrane Integrity}

Assessing cell membrane integrity is another method to measure cell viability and the induction of cytotoxic effects. Cytotoxic microparticles often compromise cell membrane integrity. 


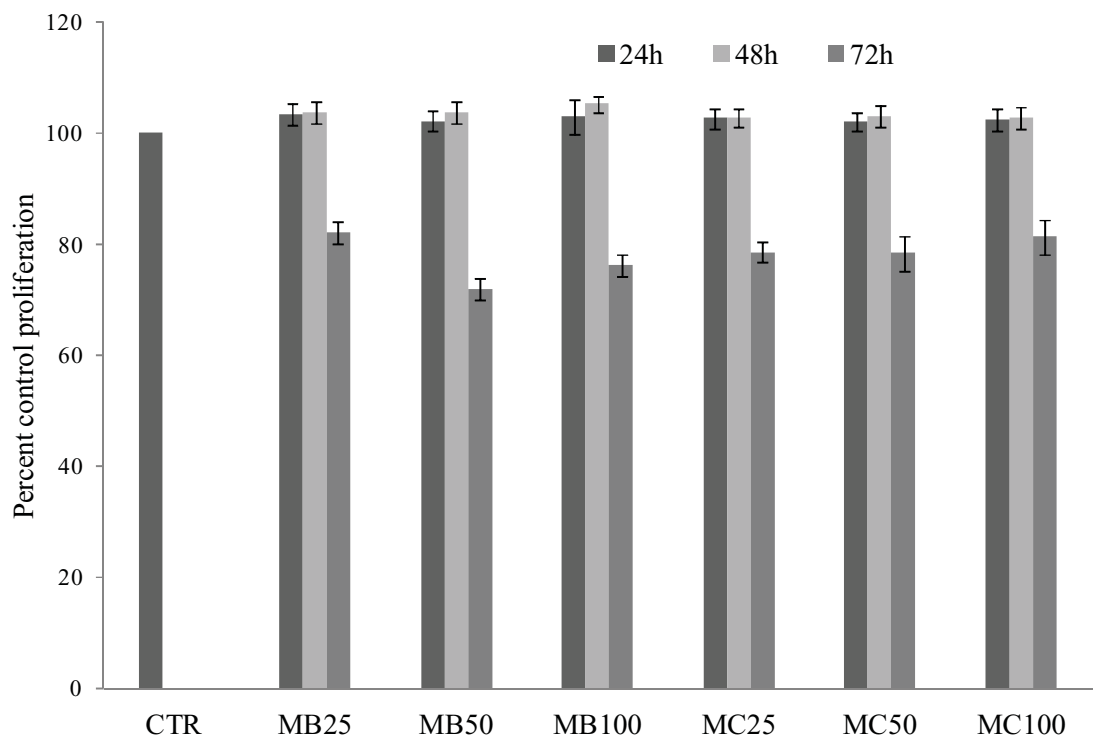

Figure 2. SKBR3 cell viability evaluation in control (CTR; valid for 24,48 , and $72 \mathrm{~h}$ ) and after incubation with LSMBs and LSMCs (25, 50100 particles/cell) for 24, 48, and $72 \mathrm{~h}$. The percent control proliferation (assayed by MTT test) was calculated considering the value of control as $100 \%$. Results are expressed as mean value \pm SD of quadruplicate determinations from three independent experiments. A significant reduction of cell viability can be noticed in $72 \mathrm{~h}$-treated samples with respect of control cells (One-way ANOVA test, $* p<0.01$ ).

Vital dyes, such as Trypan Blue (TB) or propidium iodide (PI) are usually excluded from healthy cells. Consequently to the loss of membrane integrity, TB freely crosses the membrane and stains intracellular components. SKBR3 cells were incubated with microparticles, washed once with PBS, and labeled with TB solution $(0.4 \%$ in PBS). Samples were then analyzed by flow cytometry to assess the percentage of TB-stained cells (Figure 3). Data obtained seem to indicate that the interaction between microparticles and cells up to 120 min did not induce a significant damage to the cell membrane, as demonstrated by similar percentages of TB-stained cells in control and treated samples. These findings support our previous cell viability results.

\subsection{Scanning Electron Microscopy Study}

Although it is widely recognized that the small size of nanoparticles may be beneficial for a rapid entry into cells via endocytosis, ${ }^{[40]}$ there appears to be no cut-off limit in size up to at least $5 \mu \mathrm{m}$ to gain cellular entry of microparticles through macropinocytosis. ${ }^{[41]}$ SEM was used to gain an understanding of the mechanism of interaction between cells and microparticles. Untreated SKBR3 cells displayed their typical morphology characterized by polygonal shape with numerous microvilli (Figure 4A, 5A, arrows) randomly distributed on the cell surface. After cell treatment for 10 and 30 min with LSMBs and LSMCs at the microparticles to cell ratio 20:1, numerous intact and deflated microspheres can be observed on the cell membranes (Figure 4B,C, arrows; 5B,C, arrows). In addition, a 60-min incubation induced the formation of ruffles (Figure 4D, 5D, arrows). SEM observations revealed the strong adhesion of microparticles on cell surface and confirmed the absence of cytotoxic effects along with the maintenance of cell integrity, even when numerous particles were laying on the cell surface. No evidence of cell damage could be detected even after $120 \mathrm{~min}$ of incubation (data not shown).

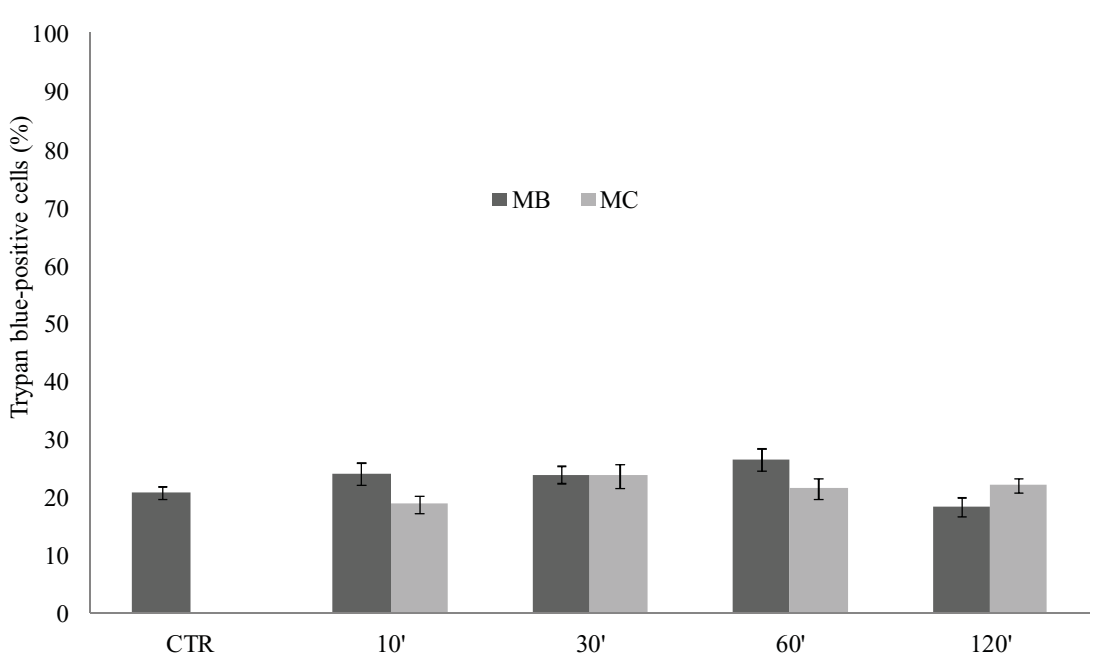

Figure 3. Analysis of cell membrane integrity by flow cytometry. SKBR3 cells were incubated for $10,30,60$, or 120 min with microparticles and then labeled with TB. Results are expressed as the mean percentage of TB-stained cells \pm SD from three independent experiments; CTR control.

\subsection{Effects of LSMB and LSMC on Cell Cycle Progression}

Cell cycle analysis was performed to investigate the effect of LSMB- and LSMC-cell interaction and particle internalization on SKBR3 proliferation. To this aim, cells were incubated with microparticles for a longer time $(24 \mathrm{~h})$ as compared with the above experiments. Samples were then fixed with ethanol and stained with PI, a specific DNA labeling dye. Cell cycle is a series of events involving cell division and duplication, comprising interphase (gap1, G1), synthesis (S), gap2 (G2), and mitosis (M) phases. Incubation with both LSMBs and LSMCs caused a slight reduction of cell number in $\mathrm{S}$ phase. In addition, LSMCs interaction induced a modest increase of $\mathrm{G} 2 / \mathrm{M}$ phase (Figure 6). This is the "DNA structure checkpoint" phase; cells accumulate at the $\mathrm{G} 2 / \mathrm{M}$ transition in response to unreplicated DNA 


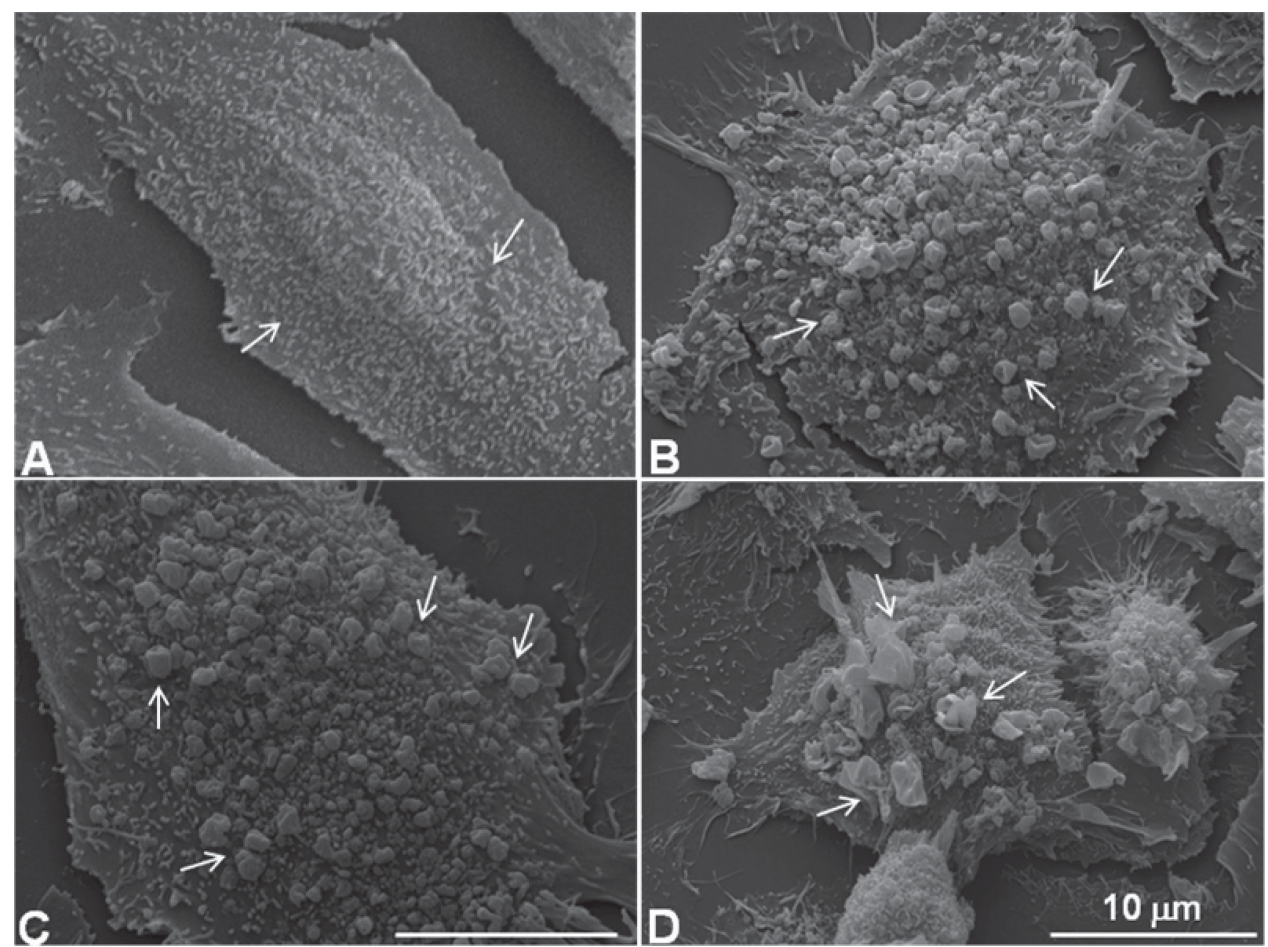

Figure 4. SEM images of SKBR3 cells after incubation with LSMBs (20:1 particles per cells). SKBR3 control cells showing normal morphology (A), after $10 \mathrm{~min}(\mathrm{~B}), 30 \mathrm{~min}$ (C; scale bar is $5 \mu \mathrm{m}$ ), and $60 \mathrm{~min}$ of incubation (D).

or DNA damage, and the "spindle assembly checkpoint" prevents anaphase until all chromosomes have obtained bipolar attachment. The combination of checkpoint deficiencies and specific types of damage would lead to mitotic catastrophe. ${ }^{[42]}$ Further experiments will be performed to better characterize the G2/M increase induced by LSMCs-cell interaction.
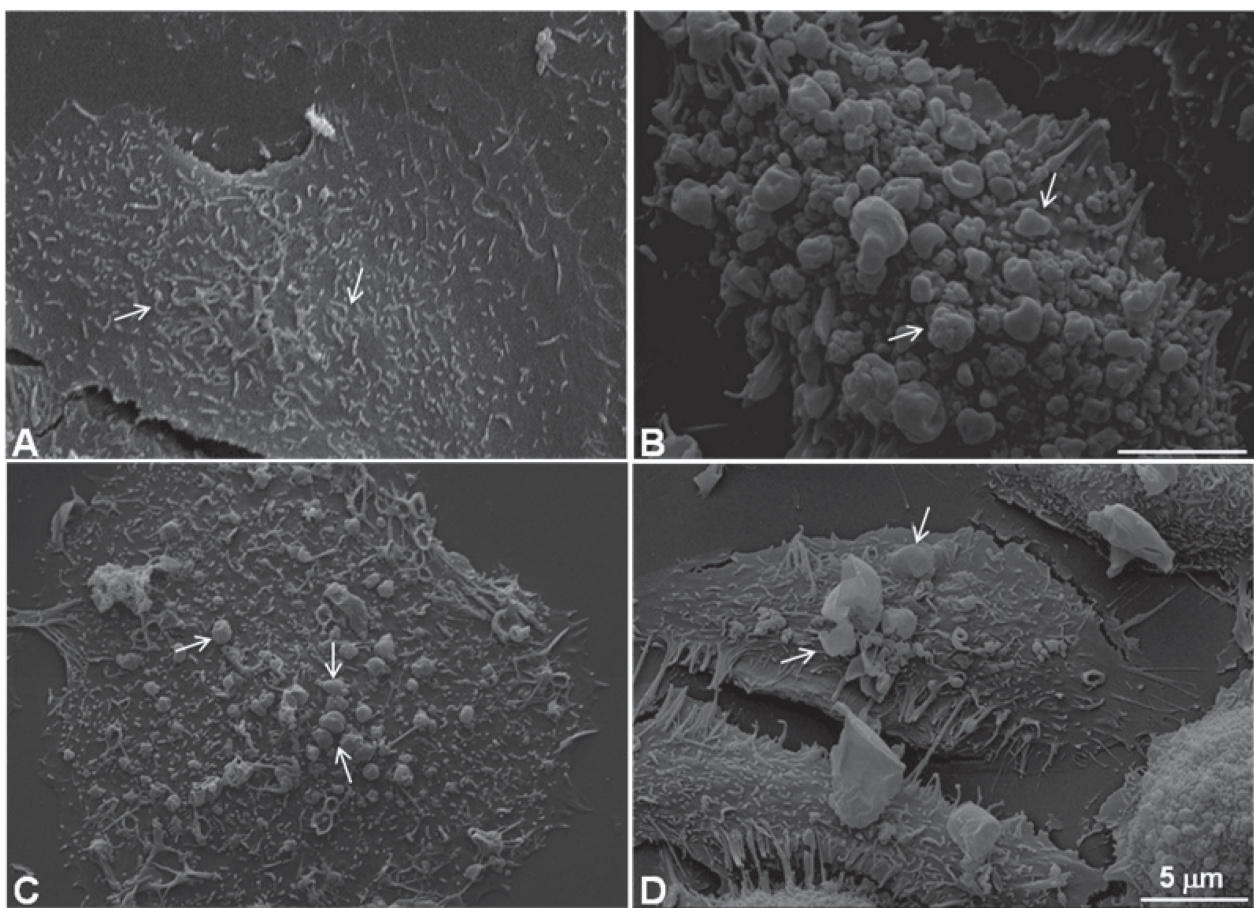

Figure 5. SEM images of SKBR3 cells after incubation LSMCs (20:1 particles per cells). SKBR3 control cells showing normal morphology (A), after $10 \mathrm{~min}(\mathrm{~B}), 30 \mathrm{~min}(\mathrm{C})$, and $60 \mathrm{~min}(\mathrm{D})$ of incubation. 

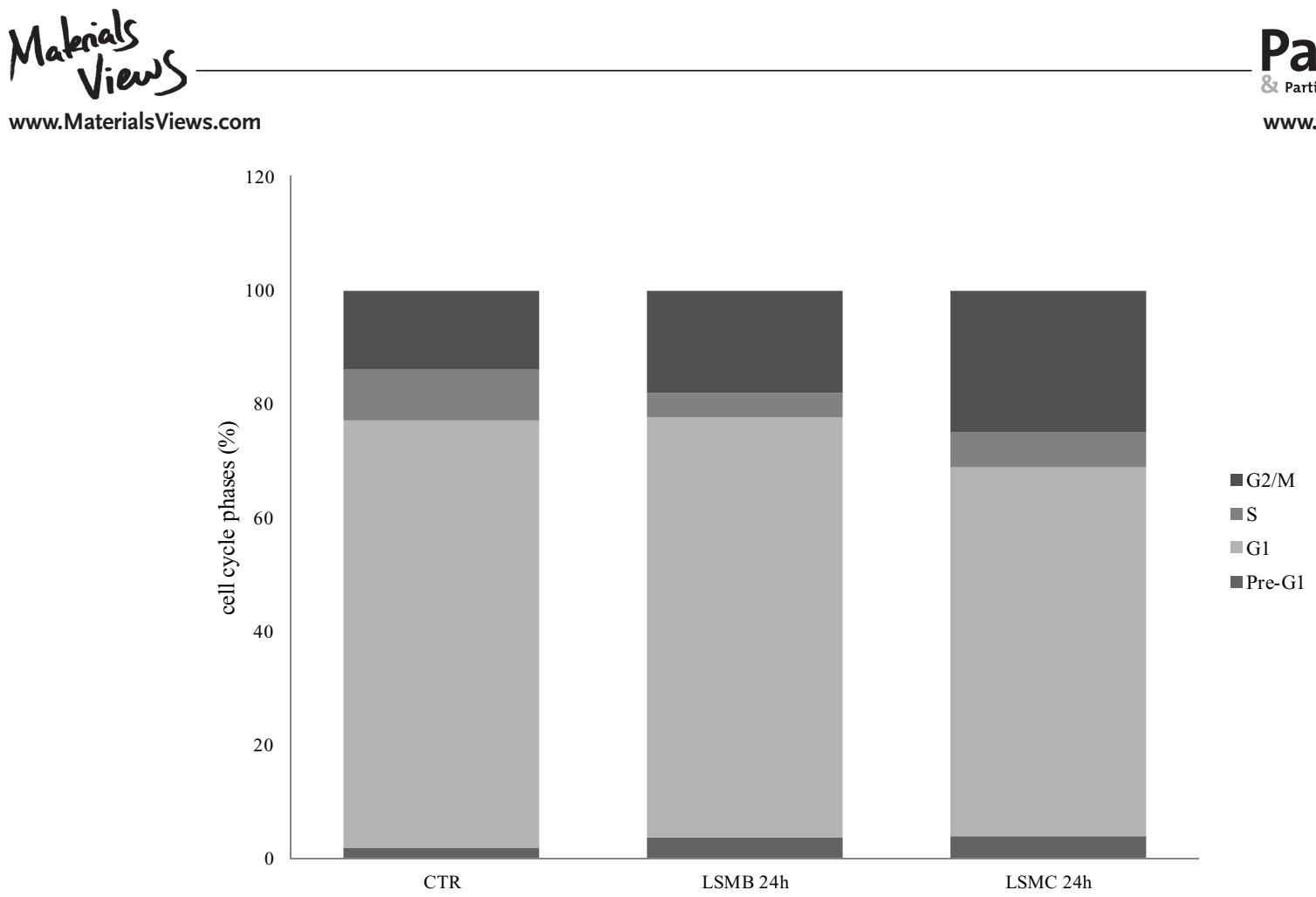

Figure 6. Cell cycle analysis by flow cytometry in control SKBR3 cells (CTR) and after incubation with LSMBs or LSMCs for 24 h. Percentages of cell cycle phases (pre-G1, G1, S, G2/M) were calculated from DNA histograms by CellQuest software.

Interestingly, microparticles did not induce the appearance of a sub-G1 population, which is considered a marker of cell death (apoptosis + necrosis) induction, thus confirming previous results on cell viability, membrane integrity, and cell morphology.

\subsection{Confocal Laser Scanning Microscopy Analysis}

To determine whether MCs were efficiently internalized by cells, their intracellular distribution was investigated by confocal laser scanning microscopy (CLSM) analysis. Fluorescently labeled LSMBs [LSMB-Fluorescein isothiocyanate isomer I (FITC)] and LSMCs (LSMC-FITC) were prepared. Cells were incubated with 20 microparticles/cell for $30 \mathrm{~min}$, $1 \mathrm{~h}$, and $2 \mathrm{~h}$. The uptake of LSMC-FITC and LSMB-FITC by SKBR3 cells was observed up to $2 \mathrm{~h}$ to obtain qualitative internalization data and also to discriminate between surfaceattached and -internalized microparticles. Figure 7A,B show internalized LSMB-FITC and LSMC-FITC, respectively, after $2 \mathrm{~h}$ of incubation. Monitoring microparticle internalization by confocal microscopy gives a more complete understanding of particle intracellular localization and degradation. While LSMC particles preserved their shape and dimension following cellular uptake (Figure 7B, arrows), LSMBs showed an altered shape and the appearance of spotty fluorescence was observed soon after the internalization phase, suggestive of early degradation (Figure 7A, arrows). To evaluate the extent of internalization, we examined a number of confocal microscopy images. By performing a manual counting of cells that internalized LSMB-FITC and LSMC-FITC particles, we obtained a percentage of $86 \pm 4$ of positive cells (total counts
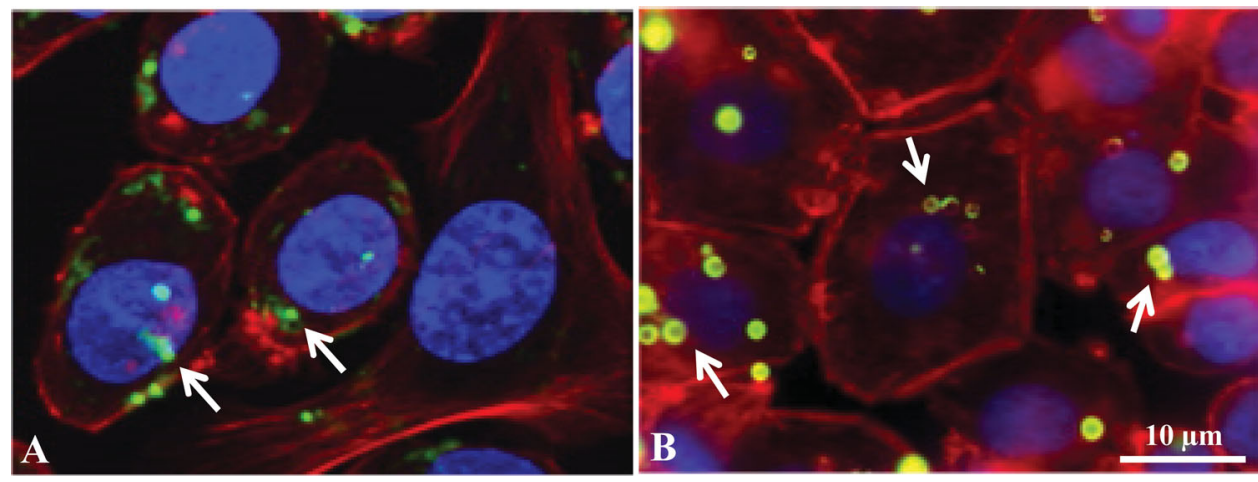

Figure 7. CLSM images of internalized microparticles. SKBR3 cells were incubated for $2 \mathrm{~h}$ with LSMB-FITC (A) and LSMC-FITC (B). Green, red, and blue indicate FITC-conjugated microparticles, actin filaments, and nuclei stained with Hoechst 33342, respectively. LSMBs and LSMCs appear to be efficiently internalized by the cells considering the low microparticles/cell ratio used for the incubation. 
$=500)$. The different morphology of internalized LSMBs and LSMCs could be related to the kinetics of their degradation (see Table 1). Hence, LSMBs are also readily hydrolyzed in the intracellular vacuole soon after the internalization, whereas the LSMCs require a longer time. In addition, no alterations of nuclear morphology could be detected in SKBR3 cells, confirming previous results on the absence of apoptosis induction by microparticles. The efficient internalization of both LSMCs and LSMBs was also confirmed by flow cytometry analysis.

\subsection{Analysis of LSMB-FITC and LSMC-FITC Cellular Uptake by Flow Cytometry}

To evaluate the efficiency and kinetics of microparticle uptake, SKBR3 cells were incubated with LSMB-FITC and LSMC-FITC using a 25 particles/cells ratio, washed, and analyzed by flow cytometry. The time course of particle internalization was studied from $10 \mathrm{~min}$ to $24 \mathrm{~h}$. The cytofluorimetric analysis demonstrated a time- and formulation-dependent uptake of the microparticles. LSMB-FITC were internalized at a higher rate than LSMC-FITC (Figure 8A). The maximum fluorescence signal from LSMB-FITC was reached at $60 \mathrm{~min}$. After $2 \mathrm{~h}$, a significant fluorescence decrease could be observed suggesting the occurrence of a degradation process. With regard to LSMC-FITC internalization, the maximum fluorescence signal was observed at $120 \mathrm{~min}$, then decreasing at $4 \mathrm{~h}$ and $24 \mathrm{~h}$. A residual presence of LSMC-FITC, either intact or fragmented, was observed after $24 \mathrm{~h}$ (Figure 8A, inset). The high fluorescence signal from LSMC-FITC detected at $4 \mathrm{~h}$ and $24 \mathrm{~h}$ suggest that these particles were degraded more slowly than LSMB-FITC, supporting results from the degradation study reported in Table 1 and microscopy observations.

In Figure 8, panel $\mathrm{B}$, representative flow cytometric profiles of control and after incubation ( 60 and $120 \mathrm{~min}$ ) samples are reported for LSMC-FITC and LSMB-FITC. It can be observed that the LSMB-treated cells histogram is characterized by a wide fluorescence profile with two populations. At $120 \mathrm{~min}$, due to the beginning of a degradation process, the first population represents almost all cells. By contrast, the fluorescent profiles of LSMC-treated cells are characterized by a time-dependent process of internalization, with an increase in signal from 60 to $120 \mathrm{~min}$ of incubation. The numbers reported above the profiles represent the internalization percentage for LSMBs (95\%) and LSMCs (98\%), showing comparable results.

We can speculate that the difference in surface chemistry observed in LSMBs and LSMCs (Table 1) may explain the different rate of internalization. Numerous studies have shown that surface properties (size, charge, shape, elasticity, and functional groups) have a significant impact on cellular internalization of a variety of nano and microcarriers. ${ }^{[34]}$

Generally, a significant diminution of particle association with the cells was observed when the particles had a negative zeta potential versus particles showing a positive zeta potential. However, both LSMCs and LSMBs have a net positive surface charge and a negative net charge after incubation with serum proteins such as albumin. This protein is the major component of fetal bovine serum (FBS) used to culture cells and it is the prominent protein adsorbed to nano-microparticles. ${ }^{[43]}$ Since SKBR3 cells were incubated with LSMCs and LSMBs in the presence of FBS, microparticles were readily coated by albumin during the incubation. Previous studies on cellular uptake of viruses and disulfide-based conjugates have shown that cell-surface associated proteins with thiol-disulfide interchange activity, such as protein disulfide isomerase, can regulate cell association. ${ }^{[44]}$

It has been shown that the introduction of thiols is associated with higher cellular uptake facilitated by thiolated surface proteins. ${ }^{[45]}$ The interaction between the exofacial thiols and thiols present on both LSMBs and LSMCs can facilitate cellmicroparticle interaction. Consequently, the faster kinetics of cell-LSMB interaction and internalization as compared with LSMCs could be explained by the significantly higher thiol concentration present on LSMB shell.

\section{Conclusion}

We have demonstrated that a different arrangement of the denatured lysozyme is possible as a function of the chemical nature of the interfaces, namely air-water or PFH-water. Consequently, the morphology of lysozyme hollow microparticles containing either air or PFH is different and have a significant influence on microparticles biofunctional properties. Antimicrobial activity against $M$. lysodeikticus, adhesion and internalization processes during incubation with SKBR3 cells seem to be controlled by the surface properties of LSMBs and LSMCs. Only LSMBs possess significant antimicrobial activity. Both LSMBs and LSMCs exhibit a strong adhesion on SKBR3 surface and an efficient internalization without inducing cytotoxic effects. The different morphologies shown by internalized LSMBs and LSMCs have been correlated to the kinetics of internalization and degradation. These results highlight the potential uses of LSMBs and LSMCs as ultrasound responsive platforms suited for biomedical and pharmaceutical applications.

\section{Experimental Section}

Material: Hen egg white lysozyme and DL-dithiothreitol (DTT) and M. lysodeikticus cells were purchased from Sigma-Aldrich. Tris(hydroxymethyl) amino-methane (Tris) was from Mallinckrodt. Milli-Q filtered water was obtained from a Millipore system (18.2 M $\Omega$ $\mathrm{cm}^{-1}$ at $25^{\circ} \mathrm{C}$ ). Perfluorohexane was purchased from Fluka. Fluorescein isothiocyanate isomer I (FITC), 5,5'-dithiobis(2-nitrobenzoic acid) (DTNB), proteinase $\mathrm{K}$ from tritirachium album were purchased from Sigma. 2,2,2-trifluoroethanol was obtained from Carlo Erba.

LSMBs and LSMCs Preparation: Lysozyme MBs and PFH-filled MCs were synthesized as described previously. ${ }^{[25,27]}$ Lysozyme $(5 \%, \mathrm{w} / \mathrm{v})$ was denatured in $50 \times 10^{-3} \mathrm{~m}$ Tris $-\mathrm{HCl}, \mathrm{pH} 8.3$, buffer by adding $3 \%$, w/v DTT and stirring for $2 \mathrm{~min}$. To synthesise PFH-filled MCs, $100 \mu \mathrm{L}$ of $\mathrm{PFH}$ was added to the solution. A 3-mm diameter microtip of a highintensity ultrasonic horn $(20 \mathrm{kHz}$, Branson) was positioned at the air-water or oil-water interface and acoustic power of $160 \mathrm{~W} \mathrm{~cm}^{-2}$ was applied for $30 \mathrm{~s}$. The mixture was then left standing for a few hours for the PFH-filled MCs to settle down and the air-filled MBs to float to the surface of the liquid. The excess DTT and the residual protein were repeatedly (5 times) washed off with Milli-Q water.

FITC Labeling of LSMBs and LSMCs: An aqueous suspension of MBs or MCs (1 mg mL $\mathrm{mb}^{-1}$ ) was fluorescently labeled with FITC in Milli-Q 


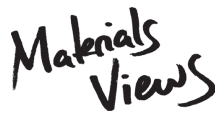

www.MaterialsViews.com
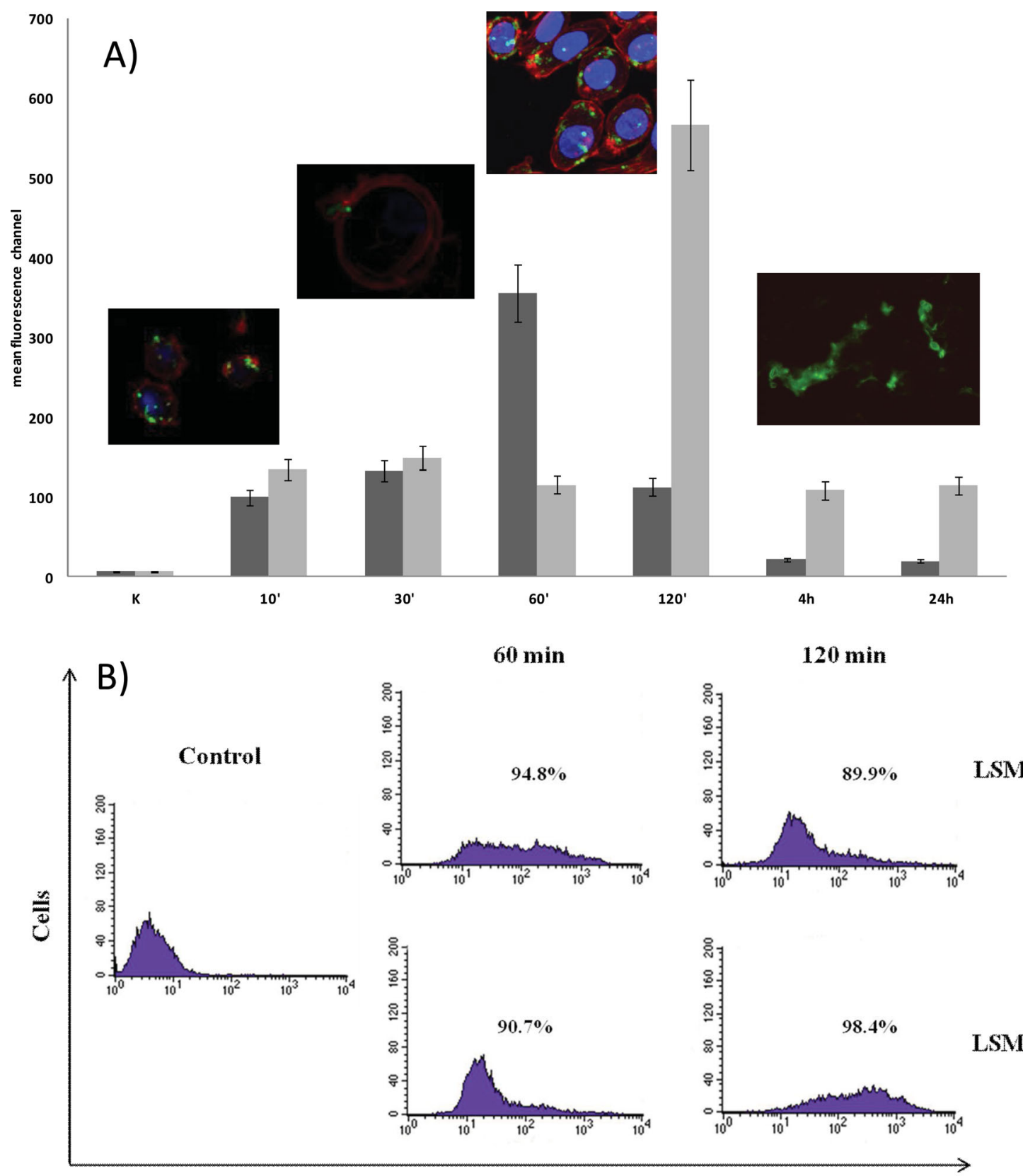

FL1-FITC

Figure 8. A) Time course analysis of LSMB-FITC (dark gray) and LSMC-FITC (gray) particles uptake performed after $10 \mathrm{~min}, 30 \mathrm{~min}, 60 \mathrm{~min}, 120 \mathrm{~min}$, $4 \mathrm{~h}$, and $24 \mathrm{~h}$; $\mathrm{K}$ - control. Fluorescence signals from microparticles were analyzed by flow cytometry and represented as mean fluorescence channel. Data represent the mean \pm the standard deviation of three independent experiments. Confocal microscopy image insets illustrate the timing of LSMB and LSMC interaction, internalization, and degradation. Particularly, at $30 \mathrm{~min}$ the formation of cellular membrane extensions surrounding the LSMB is shown. B) Flow cytometric profiles of control cells and after incubation with LSMB or LSMC for 60 and 120 min. Data are from a representative experiment. Numbers above each histogram represent the percentage of positive cells, internalized within LSMBs and LSMCs.

water. FITC-labeled MBs and MCs were separated from excess FITC by repeated washings with Milli-Q water. The concentration of MBs or MCs was determined using a microscope counting chamber emocytometer.

Microbubble and Microcapsule Characterization: An inverted Olympus IX71 wide field fluorescence microscope with a 60X objective lens was used to view the MBs and MCs. SEM (FEl Quanta) operated at an acceleration voltage of $10 \mathrm{kV}$ was used to examine the morphology and size of the MBs. SEM images were recorded on air-dried MBs sputter coated with a thin gold film. Microbubbles' average diameter and size distribution were determined over a set of 200 microparticles using optical microscope images and SEM images. The free thiol contents were determined by using Ellman's reagent, DTNB. Zeta-potential measurements were carried out on a Malvern Zetasizer. A turbidometric method was used to study the antimicrobial activity of LSMBs and LSMCs by monitoring the decrease of absorbance of a suspension of M. lysodeikticus cells at $450 \mathrm{~nm}$ as a function of time, using a 
Cary 50 Bio UV-vis spectrophotometer. The antimicrobial activity of lysozyme was assayed by the addition of $0.3 \mathrm{~mL}$ of lysozyme MBs into a $2.3 \mathrm{~mL}$ suspension of $15 \%(\mathrm{w} / \mathrm{v}) \mathrm{M}$. lysodeikticus cells in $100 \times 10^{-3} \mathrm{~m}$ phosphate buffer at $25{ }^{\circ} \mathrm{C}$ at $\mathrm{pH}$ 6.5. Time-resolved experiments were performed on a Lifespec-ps Instruments Edinburgh instrument (UK) operating in a single-photon counting mode. Fluorescence intensity decays were acquired at $340 \mathrm{~nm}$ (emission wavelength) and analyzed with the software provided by Edinburgh Instruments. The decay curves were fitted with a nonlinear least squares analysis to exponential functions through an iterative deconvolution method.

Proteolytic Degradation of Lysozyme Microbubbles and Microcapsules: Proteinase $\mathrm{K}$ was dissolved in $10 \times 10^{-3} \mathrm{~m} \mathrm{CaCl}_{2}, 50 \times 10^{-3} \mathrm{~m}$ Tris in

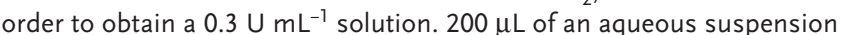
of LSMB and LSMC $\left(0.1 \mathrm{mg} \mathrm{mL}^{-1}\right)$ was incubated with $100 \mu \mathrm{L}$ of a $0.3 \mathrm{U}$ $\mathrm{mL}^{-1}$ proteinase $\mathrm{K}$ solution. The decreasing of the absorbance at $500 \mathrm{~nm}$ was monitored, using a UV-vis spectrophotometer (Varian-Cary100).

Circular Dichroism of LSMB and LSMC: CD spectra were recorded in the range $200-250 \mathrm{~nm}$ using a quartz cell $(1 \mathrm{~cm})$ on a JASCO J600 spectrometer. To a $0.2 \mathrm{mg} \mathrm{mL}^{-1}$ of LSMC suspension, an equal volume of 2,2,2-trifluoroethanol was added and the spectrum was recorded. The estimation of the percentages of protein secondary structure of $M C$ and MB was executed using the K2D and Selcom algorithm.

Cell Culture: The human breast cancer cell line SKBR3 was from American Type Culture Collection (ATCC, Rockville, MD). Cells were grown in DMEM (Euroclone) supplemented with $10 \% \mathrm{FBS}$ at $37^{\circ} \mathrm{C}$ in a humidified atmosphere containing $5 \% \mathrm{CO}_{2}$ and subcultured prior to confluence using trypsin/EDTA.

Cell Viability Assay: Cell viability was evaluated using the MTT assay. MTT assay measures the number of metabolically active cells. This parameter can be used to obtain an indication of the effects of treatments on cell viability or cell proliferation. SKBR3 cells were seeded into 96-well microtiter plates (NunclonTM, Nunc, Germany) at a density of $2 \times 10^{4}$ cells/well and grown for $24 \mathrm{~h}$. Subsequently, cells were treated with LSMBs and LSMCs at the concentrations of 25/50/100 particles/ cell. After incubation for 24,48 , and $72 \mathrm{~h}$, medium was replaced by fresh one containing $0.5 \mathrm{mg} \mathrm{mL}^{-1}$ MTT (Sigma, Deisenhofen, Germany). Unreacted dye was removed after $2 \mathrm{~h}$ of reaction at $37^{\circ} \mathrm{C}$ and the purple formazan product was dissolved in $200 \mu \mathrm{L} /$ well dimethylsulfoxide (Merck, Darmstadt, Germany). The absorbance of formazan was read at $570 \mathrm{~nm}$ on a scanning microtiter spectrophotometer plate reader. Data are expressed as absorbance values relative to untreated cells in the same experiment and standardized to $100 \%$. All experiments were performed in triplicate. ${ }^{[46]}$

Membrane Integrity Measurement: In order to evaluate the membrane integrity of SKBR3 cells after incubation with LSMBs and LSMCs (ratio $1: 20)$ at different times, the samples were stained with TB $(0.4 \%$ solution in PBS) and immediately analyzed. Fluorescence signals were evaluated with a FACScan flow cytometer (Becton Dickinson, Mountain View, CA) equipped with a $15-\mathrm{mW}, 488-\mathrm{nm}$ and air-cooled argon ion laser. The fluorescence emission was collected through a 670-nm bandpass filter and acquired in "log" mode. ${ }^{[47]}$ The percentage of TB-labeled cells was calculated by CellQuest software (Becton Dickinson).

Scanning Electron Microscopy: The effects of LSMBs and LSMCs on cell morphology were visualized by SEM. The cells were grown on glass coverslips of $12 \mathrm{~mm}$ diameter, washed in $0.2 \mathrm{~m} \mathrm{Na}$-cacodylate buffer, $\mathrm{pH} 7.4$, and fixed in $2.5 \%$ glutaraldehyde in $0.2 \mathrm{~m} \mathrm{Na}$-cacodylate buffer, $\mathrm{pH} 7.4$, for $2 \mathrm{~h}$ at room temperature. After three washes the cells were postfixed with $1 \%(\mathrm{w} / \mathrm{w}) \mathrm{OsO}_{4}$ for $1 \mathrm{~h}$, dehydrated on an ethanol gradient, critical point dried in $\mathrm{CO}_{2}$. The coverslips were attached to aluminum stubs, mounted with silver print, and coated with gold in a sputter coater. ${ }^{[48]}$ The samples were examined with a Cambridge Stereoscan 360 scanning electron microscope (Cambridge Instruments, Cambridge, UK).

Cell Cycle Analysis by Flow Cytometry: Cells grown to near confluence were treated with microparticles at $37^{\circ} \mathrm{C}$. After treatment, both floating and adherent cells were collected, washed twice with cold PBS, and centrifuged. The pellet was fixed in $70 \%$ ethanol in $\mathrm{H}_{2} \mathrm{O}$ at $4{ }^{\circ} \mathrm{C}$ for $1 \mathrm{~h}$ (overnight), washed twice with cold PBS, and then resuspended in
PBS containing $40 \mu \mathrm{g} \mathrm{mL} L^{-1} \mathrm{PI}$ and $100 \mu \mathrm{g} \mathrm{mL} \mathrm{L}^{-1}$ RNAse, at $37{ }^{\circ} \mathrm{C}$ for $1 \mathrm{~h}$. Samples were then analyzed on the FACScan flow cytometer. PI fluorescence emission was collected through a 570-nm bandpass filter. At least 10000 events/sample were acquired in a linear mode. Percentage of cells in the different phases was calculated using the CellQuest software.

Confocal Scanning Laser Microscopy: SKBR3 cells were grown on coverslips and treated with LSMB-FITC and LSMC-FITC for 4 and $24 \mathrm{~h}$. Subsequently, cells were fixed with $4 \%$ paraformaldehyde in phosphatebuffered saline (PBS, pH 7.4) for $30 \mathrm{~min}$ at room temperature and, after a washing in the same buffer, were permeabilized with $0.5 \%$ Triton X-100 (Sigma Chemicals Co., St. Louis, MO) in PBS for 5 min at room temperature. For actin detection, cells were stained with rodhaminephalloidin (Sigma) at $37{ }^{\circ} \mathrm{C}$ for $30 \mathrm{~min}$. For nuclei detection, cells were stained with Hoechst 33258 (Sigma) at $37{ }^{\circ} \mathrm{C}$ for 15 min. After washing with PBS, coverslips were mounted with glycerol-PBS (2:1) and analyzed by intensified charge-coupled device video microscopy (IVM) with a Nikon Microphot fluorescence microscope equipped with a Zeiss CCD camera. Confocal microscopy experiments were performed on an Olympus IX81 confocal laser scanning microscope equipped with laser diode 405, Ar laser $488 \mathrm{~nm}$, and HeNe laser $543 \mathrm{~nm}$.

Analysis of Cellular Uptake of LSMB-FITC and LSMC-FITC: A time course analysis of the cellular uptake of LSMBs and LSMCs was performed by flow cytometry. Microparticles were added to the culture medium at the final concentrations of $1: 25$ cell/particles at $37^{\circ} \mathrm{C}$ from $10 \mathrm{~min}$ to $24 \mathrm{~h}$. At the end of each treatment, cells were washed with ice-cold Hank's balanced salt solution (Sigma Chemical Company, St. Louis, MO), detached with EDTA and $0.25 \%$ trypsin, resuspended in ice-cold PBS and immediately analyzed. Fluorescence signals were analyzed with the FACScan flow cytometer. The fluorescence emission was collected through a 530-nm bandpass filter and acquired in "log" mode. At least 10000 events were acquired. Microparticle uptake was evaluated as fluorescence intensity, expressed as mean fluorescence channel and calculated by CellQuest software.

Statistical Analysis: One-way ANOVA test was used to perform statistical analysis of the data. Differences between two groups were considered significant at $p<0.05$.

\section{Supporting Information}

Supporting Information is available from the Wiley Online Library or from the author.

\section{Acknowledgements}

This work was supported by the University of Melbourne IPRS fellowship and Vic Govt. VIRS fellowship.

Received: January 22, 2013

Revised: March 14, 2013

Published online:

[1] R. Duncan, R. Gaspar, Mol. Pharmaceutics 2011, 8, 2101.

[2] J. Wang, J. Byrne, M. E. Napier, J. M. DeSimone, Small 2011, 7, 1919.

[3] A. Wang, R. Langer, O. Farokhzad, Annu. Rev. Med. 2012, 63, 185.

[4] R. K. Jain, T. Stylianopoulos, Nat. Rev. Clin. Oncol. 2010, 11, 653.

[5] Y. Yan, G. K. Such, A. P. R. Johnston, J. P. Best, F. Caruso, ACS Nano 2012, 6, 3663.

[6] R. Misra, S. Acharya, S. K. Sahoo, Drug Discovery Today 2010, 15, 842.

[7] Y. T. Chang, P. Y. Liao, H. S. Sheu, Y.J. Tseng, F. Y. Cheng, C. S. Yeh, Adv. Mater. 2012, 24, 3309. 
[8] I. Lentacker, B. Geers, J. Demeester, S. C. De Smedt, N. N. Sanders, Mol. Ther. 2010, 18, 101.

[9] J. Gao, H. Gu, B. Xu, Acc. Chem. Res. 2009, 42, 1097.

[10] D. Cosgrove, C. Harvey, Med. Biol. Eng. Comput. 2009, 47, 813.

[11] F. Cavalieri, M. Zhou, M. Tortora, L. Baldassarri, M. Ashokkumar, Curr. Pharm. Des. 2012, 18, 2118.

[12] F. Cavalieri, M. Zhou, M. Ashokkumar, Curr. Top. Med. Chem. 2010, 12, 1198.

[13] S. Sirsi, M. Borden, Bubble Sci. Eng. Technol. 2009, 1, 3.

[14] M. J. Blomley, T. Albrecht, D. O. Cosgrove, N. Patel, V. Jayaram, J. Butler-Barnes, R. J. Eckersley, A. Bauer, R. Schlief, Radiology 1999, $210,409$.

[15] Z. Hongjia, X. Rong, O. Qiufang, C. Lidian, D. Baowei, H. Ye, Eur. J. Rad. 2010, 73, 288

[16] K. Ferrara, R. Pollard, M. Borden, Annu. Rev. Biomed. Eng. Annu. Rev. Biomed. Eng. 2007, 9, 415

[17] G. J. Czarnota, R. Karshafian, P. N. Burns, S. Wong, A. Al Mahrouki, J. W. Lee, A. Caissie, W. Tran, C. Kim, M. Furukawa, E. Wong, A. Giles, Proc. Natl. Acad. Sci. USA 2012, 109, E2033.

[18] T. D. Tran, S. D. Caruthers, M. Hughes, J. N. Marsh, T. Cyrus, P. M. Winter, A. M. Neubauer, S. A. Wickline, G. M. Lanza, Int. J. Nanomed. 2007, 2, 515.

[19] S. A. Wickline, G. M. Lanza, Circulation 2003, 107, 1092.

[20] M. M. Kaneda, S. Caruthers, G. M. Lanza, S. A. Wickline, Ann. Biomed. Eng. 2009, 37, 1922.

[21] T. Randsoe, O. Hyldegaard, J. Appl. Physiol. 2009, 107, 1857.

[22] G. P. Giuliari, M. A. Cortez, J. Ubiera, Can. J. Ophthalmol. 2007, 42, 617.

[23] H. Nakahara, S. Lee, M. P. Krafft, O. Shibata, Langmuir 2010, 26, 18256.

[24] J. G. Riess, Tetrahedron 2002, 58, 4113.

[25] F. Cavalieri, M. Ashokkumar, F. Grieser, F. Caruso, Langmuir 2008, 24, 10078

[26] F. Cavalieri, M. Zhou, F. Caruso, M. Ashokkumar, Chem. Commun. 2012, 47, 4096

[27] M. Zhou, F. Cavalieri, M. Ashokkumar, Soft Matter 2011, 7, 623.

[28] M. Zhou, F. Cavalieri, F. Caruso, M. Ashokkumar, ACS Macro Lett. 2012, 1, 853.

[29] R. T. Bustami, H. Chan, F. Dehghani, N. R. Foster, Pharm. Res. $2000,17,1360$.
[30] A. Bouchard, N. Jovanovic, W. Jiskoot, E. Mendes, G. Witkamp, D. J. A. Crommelin, G. W. Hofland, J. Supercrit. Fluid 2007, 40, 293.

[31] P.S. Epstein, M. S. Plesset, J. Chem. Phys. 1950, 18, 1505.

[32] E. Pisani, N. Tsapis, B. Galaz, M. Santin, R. Berti, N. Taulier, E. Kurtisovski, O. Lucidarme, M. Ourevitch, B. T. Doan, J. C. Beloeil, B. Gillet, W. Urbach, S. L. Bridal, E. Fattal, Adv. Funct. Mater. 2008, 18, 2963.

[33] M. Ekkapongpisit, A. Giovia, C. Follo, G. Caputo, C. Isidoro, Int. J. Nanomed. 2012, 7, 4147.

[34] S. E. A. Gratton, P. A. Ropp, P. D. Pohlhaus, J. C. Luft, V. J. Madden, Mary, E. Napier, J. M. DeSimone, Proc. Natl. Acad. Sci. USA 2008, 105, 11613

[35] N. Doshi, S. Mitragotri, PLoS ONE 2010, 5, e10051.

[36] Y. Mine, F Ma, S Lauriau, J. Agric. Food. Chem. 2004, 52, 1088.

[37] M. Buck, Quart. Rev. Biophys. 1998, 31, 297.

[38] D. Roccatano, M. Fioroni, M. Zacharias, G. Colombo, Protein Sci. 2005, 14, 2582.

[39] M. Buck, S. E. Radford, C. M. Dobson, Biochemistry 1993, 32, 669.

[40] a) G. J. Doherty, H. T. McMahon, Annu. Rev. Biochem. 2009, 78, 857; b) K. Sandvig, S. Pust, T. Skotl, B. van Deurs, Curr. Opin. Cell Biol. 2011, 23, 413; c) G. Sahay, D. Y Alakhova, A. V Kabanov, J. Controlled Release 2010, 145, 182.

[41] H. Meng, S. Yang, Z. Li, T. Xia, J. Chen, Z. Ji, H. Zhang, X. Wang, S. Lin, C. Huang, Z. Hong Zhou, J. I. Zink, A. E. Nel, ACS Nano 2011, 5, 4434.

[42] M. Castedo, J.-L. Perfettini, T. Roumier, K. Andreau, R. Medema, G. Kroemer, Oncogene 2004, 23, 2825.

[43] M. P. Monopoli, D. Walczyk, A. Campbell, G. Elia, I. Lynch, F. B. Bombelli, K. A. Dawson, J. Am. Chem. Soc. 2011, 133, 2525.

[44] W. Sun, P. B. Davis, J. Controlled Release 2010, 146, 118.

[45] Y. Yan, Y. Wang, J. K. Heath, E. C. Nice, F. Caruso, Adv. Mater. 2011, 23, 3916.

[46] G. Arancia, A. Stringaro, P. Crateri, A. Torosantucci, C. Ramoni, F. Urbani, C. M. Ausiello, A. Cassone, Cell Immunol. 1998, 186, 28.

[47] A. Calcabrini, J. M. Garcıa-Martınez, L. Gonzalez, M. Julián Tendero, M. T. Agullo Ortuño, P. Crateri, A. Lopez-Rivas, G. Arancia, P. Gonzalez Porque, J. Martín-Pérez, Carcinogenesis 2006, 27, 1699.

[48] C. Bombelli, A. Stringaro, S. Borocci, G. Bozzuto, M. Colone, L. Giansanti, R. Sgambato, L. Toccacieli, G. Mancini, A. Molinari, Mol. Pharm. 2010, 7, 130. 